\title{
S-nitrosylation of NADPH oxidase regulates cell death in plant immunity
}

\begin{abstract}
Changes in redox status are a conspicuous feature of immune responses in a variety of eukaryotes, but the associated signalling mechanisms are not well understood. In plants, attempted microbial infection triggers the rapid synthesis of nitric oxide and a parallel accumulation of reactive oxygen intermediates, the latter generated by NADPH oxidases related to those responsible for the pathogen-activated respiratory burst in phagocytes. Both nitric oxide and reactive oxygen intermediates have been implicated in controlling the hypersensitive response, a programmed execution of plant cells at sites of attempted infection. However, the molecular mechanisms that underpin their function and coordinate their synthesis are unknown. Here we show genetic evidence that increases in cysteine thiols modified using nitric oxide, termed S-nitrosothiols, facilitate the hypersensitive response in the absence of the cell death agonist salicylic acid and the synthesis of reactive oxygen intermediates. Surprisingly, when concentrations of S-nitrosothiols were high, nitric oxide function also governed a negative feedback loop limiting the hypersensitive response, mediated by S-nitrosylation of the NADPH oxidase, AtRBOHD, at Cys 890, abolishing its ability to synthesize reactive oxygen intermediates. Accordingly, mutation of Cys 890 compromised S-nitrosothiol- mediated control of AtRBOHD activity, perturbing the magnitude of cell death development. This cysteine is evolutionarily conserved and specifically S-nitrosylated in both human and fly NADPH oxidase, suggesting that this mechanism may govern immune responses in both plants and animals.
\end{abstract}

Keyword: Plant sciences; Molecular biology; Genetics; Genomics; Chemistry 